\title{
Staircase Macdonald polynomials and the $q$-Discriminant
}

\section{Adrien Boussicault and Jean-Gabriel Luque}

Université Paris-Est, Marne-la-Vallée, Institut d'Électronique et d'Informatique Gaspard-Monge 77454 Marne-laVallée Cedex 2.

\begin{abstract}
We prove that a $q$-deformation $\mathfrak{D}_{k}(\mathbb{X} ; q)$ of the powers of the discriminant is equal, up to a normalization, to a specialization of a Macdonald polynomial indexed by a staircase partition. We investigate the expansion of $\mathfrak{D}_{k}(\mathbb{X} ; q)$ on different bases of symmetric functions. In particular, we show that its expansion on the monomial basis can be explicitly described in terms of standard tableaux and we generalize a result of King-Toumazet-Wybourne about the expansion of the $q$-discriminant on the Schur basis.

Résumé. Nous montrons qu'une $q$-déformation $\mathfrak{D}_{k}(\mathbb{X} ; q)$ des puissances du discriminant est égale, à un coefficient de normalisation près, à un polynôme de Macdonald indexé par une partition escalier pour une certaine spécialisation des paramètres. Nous examinons les développements de $\mathfrak{D}_{k}(\mathbb{X} ; q)$ dans différentes bases de fonctions symétriques. En particulier, nous montrons que son écriture dans la base des fonctions monomiales peut être explicitement décrite en terme de tableaux standard et nous généralisons un résultat de King-Toumazet-Wybourne sur le développement du $q$-discriminant dans la base de Schur.
\end{abstract}

\section{Introduction}

Let $\mathbb{X}=\left\{x_{1}, \ldots, x_{n}\right\}$ be an alphabet. The $q$-discriminant

$$
\mathfrak{D}_{1}(\mathbb{X} ; q):=\prod_{i \neq j}\left(q x_{i}-x_{j}\right),
$$

is a polynomial encountered in different fields of mathematics. In particular, its specialization at $q=1$ is the discriminant which is an example of a symmetric function invariant under the transformation $x \rightarrow x+1$ and which has been the subject of many works in invariant theory (by Cayley, Sylvester and MacMahon).

Laughlin [14] described the ground state of the electrons in the fractional Quantum Hall effect as an expression involving an even power of the Vandermonde determinant

$$
\Psi_{\text {Laughlin }}^{k}(\mathbb{X})= \pm \mathfrak{D}_{1}(\mathbb{X} ; 1)^{k} \Psi_{\text {Laughlin }}^{0}(\mathbb{X})
$$

In this paper, we give the links between the $q$-discriminant and the Macdonald polynomials. More precisely, our main result is that the "polarized powers" of the $q$-discriminant

$$
\mathfrak{D}_{k}(\mathbb{X} ; q):=\prod_{l=1}^{k} \mathfrak{D}_{1}\left(\mathbb{X} ; q^{2 l-1}\right),
$$

1365-8050 @ 2008 Discrete Mathematics and Theoretical Computer Science (DMTCS), Nancy, France 
appear when one evaluates some specialization of "staircase" Macdonald polynomials.

The powers of the discriminant $(q=1)$ are encountered also in the context of generalizations of the Selberg integral [11, 13, 27]. These integrals are closely related to the notion of Hankel hyperdeterminant [20, 21] and Jack polynomials [9, 10]. The Selberg integral admits $q$-analogue involving the $q$-discriminant (see e.g. [23] ex3 p374). It is interesting to remark that such integrals are related to Macdonald polynomials [29].

More generally, setting $t^{a} q^{b}=1$ rises deeper identities related to the generalization of the Izergin and Korepin determinant due to Gaudin [19].

The paper is organized as follow. In Section 2 , we recall notations and properties related to symmetric functions. Section 3 is devoted to the main theorem of the paper. We prove that the polynomial $\mathfrak{D}_{k}(\mathbb{X} ; q)$ is a staircase Macdonald polynomial for a specialization of the parameters $q$ and $t$. As an application, in Section 4, we give a formula for the coefficients arising in the expansion of an even power of the Vandermonde determinant in terms of monomial functions. Finally, in Section 5 , we generalize a theorem of King et al. about the expansion of the $q$-discriminant in terms of Schur functions.

\section{Background and notations}

\subsection{Symmetric functions}

We consider the $\mathbb{C}\left[\left[q, t, q^{-1}, t^{-1}\right]\right]$-algebra $S y m$ of symmetric functions over an alphabet $\mathbb{X}$, i.e. the functions which are invariant under permutations of commuting indeterminates called letters. There exists various families of such functions. We shall need the generating series of complete function:

$$
\sigma_{z}(\mathbb{X}):=\sum_{i} S^{i}(\mathbb{X}) z^{i}=\prod_{x \in \mathbb{X}} \frac{1}{1-x z}
$$

This notation is compatible with the sum $\mathbb{X}+\mathbb{Y}$ and the product $\mathbb{X} \mathbb{Y}:=\sum_{x \in \mathbb{X}, y \in \mathbb{Y}} x y$ in the following sense

$$
\sigma_{z}(\mathbb{X}+\mathbb{Y})=\sigma_{z}(\mathbb{X}) \sigma_{z}(\mathbb{Y})=\sum_{i} S^{i}(\mathbb{X}+\mathbb{Y}) z^{i}
$$

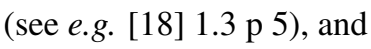

$$
\sigma_{z}(\mathbb{X} \mathbb{Y})=\sum_{i} S^{i}(\mathbb{X} \mathbb{Y}) z^{i}=\prod_{x \in \mathbb{X}} \prod_{y \in \mathbb{Y}} \frac{1}{1-x y z}
$$

(see e.g. [18] $1.5 \mathrm{p} 13$ ). In particular, if $\mathbb{X}=\mathbb{Y}$ one has $\sigma_{z}(2 \mathbb{X})=\sigma_{z}(\mathbb{X})^{2}$. This definition can be extended for any complex number $\alpha$ by putting $\sigma_{z}(\alpha \mathbb{X})=\sigma_{z}(\mathbb{X})^{\alpha}$.

We will use the Schur basis whose elements $S_{\lambda}$ are indexed by decreasing partitions and defined by

$$
S_{\lambda}:=\operatorname{det}\left(S^{\lambda_{i}-i+j}\right)_{1 \leq i, j \leq n}
$$

where $S^{i}(\mathbb{X})=0$ if $i<0$, see e.g. [23] I.3.4 p41 and [18] 1.4.2 p8. 


\subsection{Macdonald Polynomials}

The Macdonald polynomials $\left(P_{\lambda}(\mathbb{X} ; q, t)\right)_{\lambda}$ form the unique basis of symmetric functions orthogonal for the standard $q, t$ deformation of the usual scalar product on symmetric functions (see e.g. [23] VI.4 p322), verifying

$$
P_{\lambda}(\mathbb{X} ; q, t)=m_{\lambda}(\mathbb{X})+\sum_{\mu \leq \lambda} u_{\lambda \mu} m_{\mu}(\mathbb{X}) .
$$

where $m_{\lambda}$ is a monomial function in the notation of [23] I.2.1 p8. Their generating function is (see e.g. [23] VI.4.13 p324)

$$
K_{q, t}(\mathbb{X}, \mathbb{Y}):=\sigma_{1}\left(\frac{1-t}{1-q} \mathbb{X} \mathbb{Y}\right)=\sum_{\lambda} P_{\lambda}(\mathbb{X} ; q, t) Q_{\lambda}(\mathbb{Y} ; q, t),
$$

where $Q_{\lambda}(\mathbb{X} ; q, t)=b_{\lambda}(q, t) P_{\lambda}(\mathbb{X} ; q, t)$ with

$$
b_{\lambda}(q, t)=\prod_{(i, j) \in \lambda} \frac{1-q^{\lambda_{i}-j} t^{\lambda_{j}^{\prime}-i+1}}{1-q^{\lambda_{i}-j+1} t^{\lambda_{j}^{\prime}-i}},
$$

see e.g. [23] VI.6.19 p339.

Alternatively, when $\mathbb{X}=\left\{x_{1}, \ldots, x_{n}\right\}$ is a finite alphabet, the Macdonald polynomials can be defined as the eigenfunctions of the Macdonald operator $\left.{ }^{(i)}\right] \mathfrak{M}_{1}$ (see $e . g$ [23] VI.3 p315 and VI.4 p325). Indeed,

$$
P_{\lambda}(\mathbb{X} ; q, t) \mathfrak{M}_{1}=[[\lambda]]_{q, t} P_{\lambda}(\mathbb{X} ; q, t),
$$

where, for any $v \in \mathbb{N}^{n},[[v]]_{q, t}$ is defined as

$$
[[v]]_{q, t}:=q^{v_{1}} t^{n-1}+q^{v_{2}} t^{n-2}+\cdots+q^{v_{n}} .
$$

Denoting by $R(\mathbb{X}, \mathbb{Y})=\prod_{x \in \mathbb{X}, y \in \mathbb{Y}}\left(x_{i}-y_{j}\right)$ the resultant of two alphabets $\mathbb{X}$ and $\mathbb{Y}$, this operator may be defined in terms of divided differences

$$
f(\mathbb{X}) \mathfrak{M}_{1}=f\left(\mathbb{X}-(1-q) x_{1}\right) R\left(t x_{1} ; \mathbb{X}-x_{1}\right) \partial_{1} \ldots \partial_{n-1} .
$$

where, for each $i=1 \ldots n-1, \partial_{i}$, denoted on the right, is the operator (see e.g. [16])

$$
f\left(x_{1}, \ldots, x_{n}\right) \partial_{i}:=\frac{f\left(x_{1}, \ldots, x_{i}, x_{i+1}, \ldots, x_{n}\right)-f\left(x_{1}, \ldots, x_{i+1}, x_{i}, \ldots, x_{n}\right)}{x_{i}-x_{i+1}} .
$$

\section{Staircase Macdonald polynomials}

Let us denote by $\rho:=[n-1, \ldots, 1,0]$ and set $m \rho:=[m(n-1), \ldots, m, 0]$ for $m \in \mathbb{N}$. In this section, we consider a strictly positive integer $k$. We need the following lemma.

\footnotetext{
(i) Sekiguchi [26] (and later Debiard [6]) introduced a family of differentials operators depending on a parameter $\alpha$ in the context of the study of the Jack polynomials. Macdonald generalized their results by introducing $q$-analogues of these operators.
} 
Lemma 3.1 Under the specialization $t \rightarrow q^{\frac{(1-2 k)}{2}}$, the Macdonald polynomial $P_{2 k \rho}\left(\mathbb{X} ; q, q^{\frac{(1-2 k)}{2}}\right)$ belongs to an eigenspace of $\mathfrak{M}_{1}$ whose dimension is 1 and its associated eigenvalue is

$$
[[2 k \rho]]_{q, q} \frac{1-2 k}{2}=\sum_{i=1}^{n} q^{(2 k+1)(n-i) / 2} .
$$

Proof From Equation (3), the eigenvalue associated to a partition $\lambda$ is

$$
[[\lambda]]_{q, q} \frac{1-2 k}{2}=\sum_{i=1}^{n} q^{(1-2 k)(n-i) / 2+\lambda_{i}} .
$$

Then, if $[[\lambda]]_{q, q} \frac{1-2 k}{2}=[[2 k \rho]]_{q, q} \frac{1-2 k}{2}$, there exists a permutation $\sigma \in \mathfrak{S}_{n}$ such that, for each $1 \leq i \leq n$, one has $\frac{1}{2}(2 k+1)(n-\sigma(i))=\frac{1}{2}(1-2 k)(n-i)+\lambda_{i}$. It follows that

$$
\lambda_{i}-\lambda_{i+1}=\frac{1}{2}(2 k+1)(\sigma(i+1)-\sigma(i))-\frac{1}{2}(1-2 k)
$$

Since $\lambda$ is a partition and $k>-\frac{1}{2}$, one has necessarily $\lambda_{i}-\lambda_{i+1} \geq 0$ and Equality 6 implies $\sigma(i+1)-$ $\sigma(i) \geq \frac{1-2 k}{1+2 k}>-1$. This implies that $\sigma$ is the identity and $\lambda=2 k \rho$.

For simplicity, we set $p:=q^{-\frac{1}{2}}$ and we will consider a finite alphabet $\mathbb{X}=\left\{x_{1}, \cdots, x_{n}\right\}$. Our main result is that the polarized powers $\mathfrak{D}_{k}(\mathbb{X}, p)$ of the discriminant are staircase Macdonald polynomials for the specialization considered here.

Theorem 3.2 One has

$$
\mathfrak{D}_{k}(\mathbb{X} ; p)=(-p)^{\frac{1}{2} k^{2} n(n-1)} P_{2 k \rho}\left(\mathbb{X} ; q, p^{2 k-1}\right) .
$$

Proof Reordering factors in $\mathfrak{D}_{k}\left(q x_{1}, x_{2}, \ldots, x_{n} ; p\right) R\left(p^{2 k-1} x_{1} ; \mathbb{X}-x_{1}\right)$, one obtains

$$
\mathfrak{D}_{k}\left(q x_{1}, x_{2}, \ldots, x_{n} ; p\right) R\left(p^{2 k-1} x_{1} ; \mathbb{X}-x_{1}\right) \quad=\quad \mathfrak{D}_{k}(\mathbb{X} ; p) R\left(p^{-(2 k+1)} x_{1} ; \mathbb{X} \quad-\quad x_{1}\right)
$$

Hence, applying Equation $(8)$, the polynomial $\mathfrak{D}_{k}(\mathbb{X} ; p) \mathfrak{M}_{1}$ can be rewritten as

$$
\mathfrak{D}_{k}(\mathbb{X} ; p) \mathfrak{M}_{1}=\mathfrak{D}_{k}(\mathbb{X} ; p) R\left(p^{-2 k-1} x_{1} ; \mathbb{X}-x_{1}\right) \partial_{1} \cdots \partial_{n-1}
$$

Since the polynomial $\mathfrak{D}_{k}(\mathbb{X} ; p)$ is symmetric in $\mathbb{X}$, it commutes with $\partial_{1}, \ldots, \partial_{n-1}$ and then

$$
\mathfrak{D}_{k}(\mathbb{X} ; p) R\left(p^{-2 k-1} x_{1} ; \mathbb{X}-x_{1}\right) \partial_{1} \cdots \partial_{n-1}=R\left(p^{-2 k-1} x_{1} ; \mathbb{X}-x_{1}\right) \partial_{1} \cdots \partial_{n-1} \mathfrak{D}_{k}(\mathbb{X} ; p)
$$

The remaining factor $R\left(p^{-2 k-1} x_{1} ; \mathbb{X}-x_{1}\right)$ is of total degree $n-1$ and therefore is sent to a constant $\mathbb{k}$ under $\partial_{1} \ldots \partial_{n-1}$. We use the following lemma to compute this constant.

Lemma 3.3 For any letters $a, b$,

$$
R\left(a x_{1} ; b x_{2}, \cdots, b x_{n}\right) \partial_{1} \cdots \partial_{n-1}=\sum_{i+j=n-1} a^{i} b^{j} .
$$


Proof Rewrite $R\left(a x_{1} ; b x_{2}, \ldots, b x_{n}\right)$ as

$$
S_{n-1}\left(a x_{1}-b\left(\mathbb{X}-x_{1}\right)\right)=S_{n-1}\left((a+b) x_{1}-b \mathbb{X}\right)=\sum x_{1}^{i} S_{i}(a+b) S_{n-1-i}(-b \mathbb{X}) .
$$

The image of this sum under $\partial_{1} \ldots \partial_{n-1}$ is $S_{n-1}(a+b) S_{0}(-b \mathbb{X})$ as wanted.

Applying Lemma 3.3 , one obtains the value of $\mathbb{k}$,

$$
\mathbb{k}=\sum_{i=1}^{n} p^{(2 k+1)(i-n)}
$$

From Equality $\left[5\right.$, since $p=q^{-\frac{1}{2}}$, one recognizes that $\mathbb{k}=[|2 k \rho|]_{q, p^{2 k-1}}$. This shows that

$$
\mathfrak{D}_{k}(\mathbb{X} ; p)=\beta_{k, n}(p) P_{2 k \rho}\left(\mathbb{X} ; q, p^{2 k-1}\right),
$$

where $\beta_{k, n}(p)$ is a constant depending only on $p, k$ and $n$. It remains to compute the coefficient $\beta_{k, n}(p)$. Since we know that the dominant coefficient in $P_{2 k \rho}\left(\mathbb{X} ; q, p^{2 k-1}\right)$ is 1 by definition, it suffices to compute the coefficient of the monomial $x_{n}^{2 k(n-1)} \cdots x_{2}^{2 k}$ in $\mathfrak{D}_{k}(\mathbb{X}, p)$. One finds

$$
\beta_{k, n}(p)=(-p)^{\frac{1}{2} k^{2} n(n-1)} \text {. }
$$

This ends the proof. $\square$

Example 3.4 For $k=2$ and $n=4$, one obtains

$$
P_{[12840]}\left(x_{1}+x_{2}+x_{3}+x_{4} ; q, q^{-3 / 2}\right)=q^{12} \prod_{i \neq j}\left(\left(q^{-\frac{1}{2}} x_{i}-x_{j}\right)\left(q^{-\frac{3}{2}} x_{i}-x_{j}\right)\right)
$$

\section{Expansion of Macdonald polynomials in terms of monomial func- tions}

Macdonald gives in [23] VI.7.10 p345 the following expansion of the polynomials $Q_{\lambda}$ in terms of monomial functions:

$$
Q_{\lambda}(\mathbb{X} ; q, t)=\sum_{\mu}\left(\sum_{T} \phi_{T}(q, t)\right) m_{\mu}(\mathbb{X}),
$$

where the inner sum is over the tableaux of shape $\lambda$ and evaluation $\mu$ and each $\phi_{T}(q, t)$ is an explicit rational function given in [23] VI.7.11 p346.

Theorem 3.2 and Equality 12 furnish an expansion of $\mathfrak{D}_{k}(\mathbb{X} ; p)$ according to the monomial basis,

$$
\mathfrak{D}_{k}(\mathbb{X} ; p)=\frac{(-p)^{\frac{1}{2} k^{2} n(n-1)}}{b_{2 k \rho}\left(q, p^{2 k-1}\right)} \sum_{\lambda}\left(\sum_{T} \phi_{T}\left(q, p^{2 k-1}\right)\right) m_{\lambda}(\mathbb{X})
$$

where the inner sum is over the tableaux of shape $2 k \rho$ and evaluation $\lambda$. 
Recall that Jack polynomials [9, 10] $P_{\lambda}^{(\alpha)}(\mathbb{X})$ are obtained from $P_{\lambda}(\mathbb{X} ; q, t)$ setting $q=t^{\alpha}$ and taking the limit when $t$ tends to 1 (see [23] VI 10). One has

$$
P_{\lambda}^{(\alpha)}(\mathbb{X})=\lim _{t \rightarrow 1} P_{\lambda}\left(\mathbb{X} ; t^{\alpha}, t\right), \text { and } Q_{\lambda}^{(\alpha)}(\mathbb{X})=\lim _{t \rightarrow 1} Q_{\lambda}\left(\mathbb{X} ; t^{\alpha}, t\right)=b_{\lambda}^{(\alpha)} P_{\lambda}^{(\alpha)}
$$

where $b_{\lambda}^{(\alpha)}:=\lim _{t \rightarrow 1} b_{\lambda}\left(t^{\alpha}, t\right)$. Putting

$$
\phi_{T}^{(\alpha)}:=\lim _{t \rightarrow 1} \phi_{T}\left(t^{\alpha}, t\right),
$$

one get from Equation (13) an expansion of integral powers of the discriminant.

\section{Corollary 4.1 One has}

$$
\begin{aligned}
\mathfrak{D}_{1}(\mathbb{X} ; 1)^{k}=\mathfrak{D}_{k}(\mathbb{X} ; 1) & =(-1)^{\frac{k n(n-1)}{2}} P_{2 k \rho}^{\left(\alpha_{k}\right)}(\mathbb{X}) \\
& =(-1)^{\frac{k n(n-1)}{2}}\left(b_{2 k \rho}^{\left(\alpha_{k}\right)}\right)^{-1} \sum_{\lambda}\left(\sum_{T} \phi_{T}^{\left(\alpha_{k}\right)}\right) m_{\lambda},
\end{aligned}
$$

where $\alpha_{k}=\frac{-2}{2 k-1}$ and the inner sum is over the tableaux of shape $2 k \rho$ and evaluation $\lambda$.

Example 4.2 Consider an alphabet $\mathbb{X}=\left\{x_{1}, x_{2}, x_{3}\right\}$ of size 3 . One has,

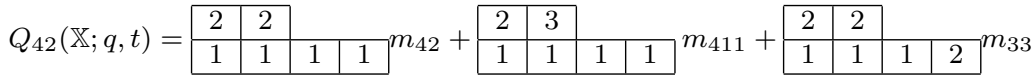

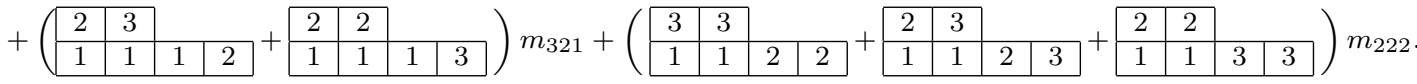

Each tableau $T$ is interpreted as the function $\Phi_{T}$,

$$
\begin{aligned}
Q_{42}(\mathbb{X} ; q, t)= & \left(\frac{1-t}{1-q}\right)^{2}\left(\frac{1-t q}{1-q^{2}}\right)^{2}\left(\frac{1-t^{2} q^{2}}{1-t q^{3}}\right)\left(\frac{1-t^{2} q^{3}}{1-t q^{4}}\right) m_{42}+ \\
& \left(\frac{1-t}{1-q}\right)^{3}\left(\frac{1-t q}{1-q^{2}}\right)\left(\frac{1-t^{2} q^{3}}{1-t q^{4}}\right)\left(\frac{1-t^{2} q^{2}}{1-q^{3} t}\right) m_{411}+\ldots
\end{aligned}
$$

Setting $q=t^{-2}$ and taking the limit $t \rightarrow 1$, the algorithm described here allows one to compute the expansion of the Jack polynomials according to the monomial functions. After simplification, one obtains

$$
Q_{42}^{(-2)}(\mathbb{X})=\frac{1}{280} m_{4,2}-\frac{1}{140} m_{4,1,1}-\frac{1}{140} m_{3,3}+\frac{1}{140} m_{3,2,1}-\frac{3}{140} m_{2,2,2} .
$$

And finally, $\mathfrak{D}_{1}(\mathbb{X} ; 1)=-m_{4,2}+2 m_{4,1,1}+2 m_{3,3}-2 m_{3,2,1}+6 m_{2,2,2}$.

Corollary 4.1 can be applied to expand Hankel hyperdeterminants. Hyperdeterminants are polynomials defined by Cayley in the aim of generalizing the notion of determinant to higher dimensional arrays (ii) [4, 5]. Given a $m$ th order tensor $\mathrm{M}=\left(M_{i_{1} \ldots i_{m}}\right)_{1 \leq i_{1}, \ldots, i_{m} \leq n}$ on a $n$ dimensional space, its hyperdeterminant is

$$
\operatorname{Det}(\mathrm{M})=\frac{1}{n !} \sum_{\sigma_{1}, \ldots, \sigma_{m} \in \mathfrak{S}_{n}} \operatorname{sign}\left(\sigma_{1} \ldots \sigma_{m}\right) \prod_{i=1}^{m} M_{\sigma_{1}(i) \ldots \sigma_{m}(i)} .
$$

(ii) Note that Cayley proposed several generalizations of determinants. The polynomial considered here is the simplest one in the sense that it generalizes the expansion of a determinant as an alternated sum. Reader can refer to [20, 21, 22, 24, 28] for more informations on the subject. 
Note that this polynomial vanishes when $m$ is odd. Suppose that $m=2 k$ is an even integer. An Hankel hyperdeterminant is an hyperdeterminant whose entries depend only on the sum of the indices $M_{i_{1} \ldots i_{2 k}}=f\left(i_{1}+\cdots+i_{2 k}\right)$. This kind of hyperdeterminant has been already considered by the authors in collaboration with Thibon and Belbachir [20, 21, 2]. In particular, it is shown that the coefficients $C_{\lambda}(n, l)$ arising in the expression

$$
\operatorname{Det}\left(M_{i_{1}+\cdots+i_{2 k}}\right)=\sum_{\lambda} C_{\lambda}(n, k) \prod_{i=1}^{n} f\left(\lambda_{i}\right),
$$

are equal (up to a multiplicative term equal to the number of permutations of $\lambda$ divided by $n$ !) to those arising in the expansion of $\mathfrak{D}_{k}(\mathbb{X} ; 1)$ in terms of monomial functions.

Example 4.3 From the expansion of the Jack polynomial $P_{84}^{(-2 / 3)}$, for an alphabet of size 3 ,

$$
\begin{aligned}
P_{84}^{(-2 / 3)}\left(x_{1}+x_{2}+x_{3}\right)= & m_{84}-4 m_{831}+6 m_{822}-4 m_{75}+12 m_{741}-8 m_{732}+6 m_{66}-8 m_{651} \\
& -22 m_{642}+48 m_{633}+48 m_{552}-36 m_{543}+90 m_{44},
\end{aligned}
$$

one deduces the expansion of the Hankel hyperdeterminant

$$
\begin{aligned}
\operatorname{Det}\left(f\left(i_{1}+i_{2}+i_{3}+i_{4}\right)\right)_{0 \leq i_{1}, i_{2}, i_{3}, i_{4} \leq 3}= & f(8) f(4) f(0)-4 f(8) f(3) f(1)+3 f(8) f(2)^{2} \\
& -4 f(7) f(5) f(0)+12 f(7) f(4) f(1)-8 f(7) f(3) f(2) \\
& +3 f(6)^{2} f(0)-8 f(6) f(5) f(1)-22 f(6) f(4) f(2) \\
& +24 f(6) f(3)^{2}+24 f(5)^{2} f(2)-36 f(5) f(4) f(3) \\
& +15 f(4)^{3} .
\end{aligned}
$$

Furthermore, in [16] Lapointe et al. gave a determinantal expression of Jack polynomial in terms of monomial functions. These computations leads naturally to a determinantal expression for Hankel hyperdeterminants.

Note that the formula for the Macdonald polynomials $\tilde{H}_{\lambda}$, given by Haglund, Haiman and Loehr [8], provides an expansion of $\mathfrak{D}_{k}(\mathbb{X} ; q)$ in terms of modified monomial functions $m_{\lambda}(\mathbb{X}(1-t))$ having a combinatorial interpretation.

\section{Expansion of the polarized powers of the $q$-discriminant in terms of Schur functions}

Di Francesco et al. [7] considered the problem of the expansion of the discriminant in terms of Schur functions. They defined the $n$-admissible partitions to be the partitions in the interval $\left[(n-1)^{n}\right],[2(n-$ $1), \ldots, 2,0]$ (with respect to the dominance order). They conjectured that they are exactly those occurring in the expansion of the discriminant. This conjecture is false as shown by Scharf et al. [25]. However, King et al. [12] proved that it becomes true when replacing the discriminant by the $q$-discriminant.

In this section, we generalize this property to $\mathfrak{D}_{k}(\mathbb{X} ; q)$. We define $(n, m)$-admissible partitions to be the partitions which appear in the expansion

$$
m_{\rho}(\mathbb{X})^{m-1} S_{\rho}(\mathbb{X})=\sum_{\lambda} b_{\lambda}^{n, m} m_{\lambda}(\mathbb{X})
$$


where $\mathbb{X}$ is an alphabet of size $n$. When $m=2 k$ is even, the $(n, 2 k)$-admissible partitions are those of the interval $\left[(k(n-1))^{n}\right],[2 k(n-1), \ldots, 2 k, 0]$. We prove that a partition appear in the expansion of $\mathfrak{D}_{k}(\mathbb{X} ; q)$ in terms of Schur functions if and only if it is a $(n, 2 k)$-partition.

\subsection{Computing admissible partitions}

Let us denote by $A_{n, m}$ the set defined recursively by

$$
\begin{aligned}
& A_{n, 1}:=\left\{\lambda=\left[\lambda_{1}, \ldots, \lambda_{n}\right] \mid \rho \geq \lambda\right\} \\
& A_{n, m}:=\left\{\left(\left(\lambda_{1}+\sigma(1)-1, \ldots, \lambda_{n}+\sigma(n)-1\right)\right) \mid \sigma \in \mathfrak{S}_{n} \text { and } \lambda \in A_{n, m-1}\right\},
\end{aligned}
$$

where $\left(\left(\mu_{1}, \ldots, \mu_{n}\right)\right)$ is the only decreasing vector obtained by reordering the components of the vector $\left[\mu_{1}, \ldots, \mu_{n}\right] \in \mathbb{R}^{n}$.

Lemma 5.1 Let $\lambda$ be a partition. The following assertions are equivalent.

1. The partition $\lambda$ belongs to $A_{n, m}$.

2. The partition $\lambda$ is $(n, m)$-admissible.

3. $\lambda$ is a partition with $n$ parts (eventually, some of theim equal zero (iii) less or equal to m $\rho$ with respect to the dominance order.

Proof The equivalence between the assertions 1 and 2 is straightforward from Equations 16 and 17 . Furthermore, from Equation (17), the maximal partition of $A_{n, m}$ is $m \rho$. It remains to prove $3 \Rightarrow 1$. We proceed by induction on $m$, if $m=1$ then the result is trivial. Suppose that $m>1$. Let $\lambda$ be a partition with $n$ parts (a part may equal zero) less or equal to $m \rho$ with respect to the dominance order. Then $((\lambda-\rho))$ is a partition less or equal to $(m-1) \rho$. Indeed, putting $((\lambda-\rho))=\left(\mu_{1}, \ldots, \mu_{n}\right)$, for a permutation $\sigma \in \mathfrak{S}_{n}$, one has $\mu_{i}=\lambda_{\sigma(i)}+n-\sigma(i)$. Hence, for each $i$

$$
\begin{aligned}
\mu_{1}+\cdots+\mu_{i} & \leq \lambda_{\sigma(1)}+\cdots+\lambda_{\sigma(i)}+n-\sigma(1)+\cdots+n-\sigma(i) \\
& \leq \lambda_{1}+\cdots+\lambda_{i}+n-1+\cdots+n-i \\
& \leq(m-1)\left(n-\frac{1}{2} i(i+1)\right)
\end{aligned}
$$

implies $((\lambda-\rho)) \leq(m-1) \rho$ for the dominance order.

By induction, $((\lambda-\rho))$ belongs to $A_{n, m-1}$. Furthermore, there exists a permutation $\sigma$ such that $((\lambda-\rho))+\rho^{\sigma}=\lambda$. Hence, from Equation 17), $\lambda \in A_{n, m} . \square$

\subsection{Counting admissible partitions}

One considers the free commutative monoid $\mathfrak{T}$ generated by the symbols $T=\left\{\tau_{1}, \ldots, \tau_{n-1}\right\}$ acting on the vectors of size $n$ by

$$
\tau_{i}\left[v_{1}, \ldots, v_{n}\right]=\left[v_{1}, \ldots, v_{i-1}, v_{i}-1, v_{i+1}+1, v_{i+1}, \ldots, v_{n}\right] .
$$

For a given vector $v \in \mathbb{Z}^{n}, \mathfrak{T} . v$ is the set of vectors $w=\left[w_{1}, \ldots, w_{n}\right] \in \mathbb{Z}^{n}$ of same weight (i.e. $v_{1}+\cdots+v_{n}=w_{1}+\ldots w_{n}$ ) lower or equal to $v$ for the dominance order. In particular, if $v=\lambda$ is

(iii) One considers partitions which contain eventually some parts equal to 0. Hence, partitions with less than $n$ parts are completed to $n$ parts by adding 0 . 
a partition then $\mathfrak{T} . \lambda$ contains all the partition of size $n$ lower or equal to $\lambda$. To each vector $v \in \mathbb{Z}^{n}$, one associates the monomial $z^{v}=z_{1}^{v_{1}-v_{2}} \ldots z_{n-1}^{v_{n-1}-v_{n}}$. For a given weight, the monomial $z^{v}$ characterizes completely $v$, furthermore $v$ is a (decreasing) partition if and only if its weight is non negative and the degree of the monomial $z^{v}$ in each variable $z_{i}$ is non-negative.

Example 5.2

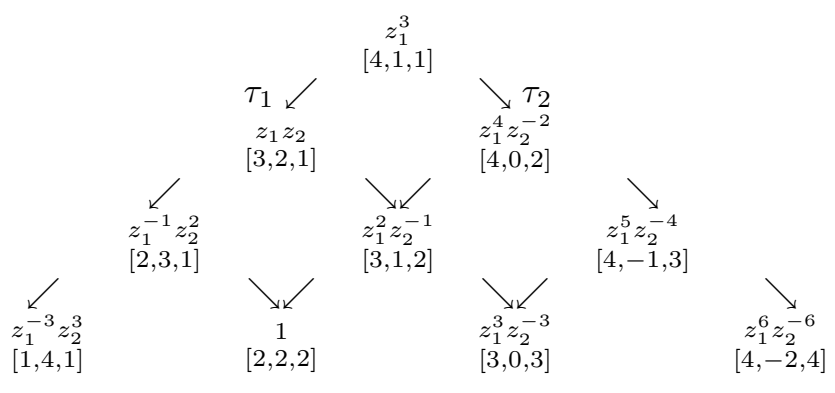

Acting on $v$ by $\tau_{i}$ is equivalent to multiply $z^{v}$ by

$$
t_{i}= \begin{cases}\frac{z_{i-1} z_{i+1}}{z_{i}^{2}} & \text { if } 1<i<n-1 \\ \frac{z_{2}}{z_{1}^{2}} & \text { if } i=1 \\ \frac{z_{n-2}^{2}}{z_{n-1}^{2}} & \text { if } i=n-1 .\end{cases}
$$

Since there are no algebraic relations between the $t_{i}^{\prime} s$, each vector appears in $\mathfrak{T} . v$ with multiplicity 0 or 1 . In other words, one has

$$
\sigma_{q}(T) \cdot z^{v}=\prod_{i} \frac{1}{1-t_{i} q} \cdot z^{v}=\sum_{w \leq v} q^{\alpha_{v, w}} z^{w} .
$$

where $\alpha_{v, w}$ is the degree of the monomial acting on $v$ to obtain $w$. Extracting the monomial which encodes a partition is equivalent to extract the part of the series (19) constituted only with non-negative exponents. This operation is performed by the MacMahon Omega operator (see e.g [1])

$$
\Omega_{x_{1}, \ldots, x_{p}} \sum_{n_{1}, \ldots, n_{p} \in \mathbb{Z}} \alpha_{n_{1}, \ldots, n_{p}} x_{1}^{n_{1}} \ldots . x_{p}^{n_{p}}=\sum_{n_{1}, \ldots, n_{p} \in \mathbb{N}} \alpha_{n_{1}, \ldots, n_{p}} x_{1}^{n_{1}} \ldots \ldots x_{p}^{n_{p}} .
$$

Example 5.3 One has

$$
\Omega_{z_{1}, z_{2}} \frac{z_{1}^{3}}{\left(1-\frac{z_{1}}{z_{2}^{2}} q\right)\left(1-\frac{z_{2}}{z_{1}^{2}} q\right)}=z_{1}^{3}+q z_{1} z_{2}+q^{3}
$$

which implies that the set of the partitions of size 3 lower or equal to [411] is $\{[411],[321],[222]\}$.

Hence,

Proposition 5.4 The size $n \geq 2$ of the alphabet being fixed, the generating series of the $(n, k)$-admissible partitions is the rational function

$\mathfrak{A}_{n}\left(q, t ; z_{1}, \ldots, z_{n-1}\right)=\Omega_{z_{1}, \ldots, z_{n-1}}\left(\left(1-t z_{1} \ldots z_{n-1}\right)\left(1-\frac{z_{0} z_{2}}{z_{1}^{2}} q\right)\left(1-\frac{z_{1} z_{3}}{z_{2}^{2}} q\right) \ldots\left(1-\frac{z_{n-2} z_{n}}{z_{n-1}^{2}} q\right)\right)^{-1}$,

where $z_{0}=z_{n}=1$. 


\subsection{Characterization of the partitions arising in the expansion of $\mathfrak{D}_{k}(\mathbb{X} ; q)$}

In this subsection, one extends the result of King-Toumazet-Wybourne to the polynomials $\mathfrak{D}_{k}(\mathbb{X} ; q)$.

Theorem 5.5 Expand $\mathfrak{D}_{k}(\mathbb{X} ; q)$ in terms of Schur functions,

$$
\mathfrak{D}_{k}(\mathbb{X} ; q)=\sum_{\lambda} c_{\lambda}(q) S_{\lambda}(\mathbb{X})
$$

Then, $c_{\lambda}(q) \neq 0$ if and only if $\lambda$ is a $(n, 2 k)$-admissible partition.

Proof Let us prove first the only if part. From Theorem 3.2 , the polynomial $\mathfrak{D}_{k}(\mathbb{X} ; q)$ equals (up to a multiplicative coefficient) a specialization of the Macdonald $P_{2 k \rho}(\mathbb{X} ; q, t)$. But it is well known that the partitions arising in the expansion of $P_{2 k \rho}(\mathbb{X} ; q, t)$ in terms of Schur functions belong to the interval $\left[(k(n-1))^{n}\right], 2 k \rho$ (see $e . g$. the determinantal expression of Macdonald polynomials given in [16]). From Lemma 5.1, this is equivalent to the fact that $\lambda$ is $(n, 2 k)$-admissible.

Conversely, to prove that the admissibility of $\lambda$ implies the non nullity of $c_{\lambda}(q)$, it suffices to prove it for a specialization. We will set $q=-1$. In this case,

$$
\mathfrak{D}_{k}(\mathbb{X} ;-1)=\prod_{i \neq j}\left(x_{i}+x_{j}\right)^{k}=S_{\rho}(\mathbb{X})^{2 k} .
$$

We will prove a stronger result showing that the coefficient $c_{\lambda}^{n, m}$ in the expansion

$$
S_{\rho}(\mathbb{X})^{m}=\sum_{\lambda} c_{\lambda}^{n, m} S_{\lambda}(\mathbb{X})
$$

is non-zero if and only if $\lambda$ is $(n, m)$-admissible. We proceed by induction on $m$. Note that the initial case $(m=2)$ has been proved by King-Toumazet-Wybourne in [12] Corollary 3.2 as a consequence of an important result of Berenstein-Zelevinsky [3].

One needs the two following lemmas

Lemma 5.6 If $\lambda$ is a $(n, m)$-admissible partition $(m>1)$, then $((\lambda-\rho))$ is a $(n, m-1)$-admissible partition.

Proof From Equality 16 , each $(n, m)$-admissible partition can be obtained by adding a permutation of $\rho$ to a $(n, m-1)$-admissible partition. Our statement is a consequence of this fact.

Lemma 5.7 Let $\mu \subset \lambda$ be a partition and $\nu:=\left(\left(\lambda_{1}-\mu_{1}, \ldots, \lambda_{n-1}-\mu_{n-1}, \lambda_{n}-\mu_{n}\right)\right)$. Then, the Littlewood-Richardson coefficient $c_{\mu \nu}^{\lambda}=\left\langle S_{\lambda}, S_{\mu} S_{\nu}\right\rangle$ equals 1 .

Proof The Littlewood-Richardson coefficient $c_{\mu \nu}^{\lambda}$ is equal to the number of tableaux of shape $\nu$ and evaluation $\lambda-\mu$. But $\lambda-\mu$ is a permutation of $\nu$ and Theorem 11.4.3 of [18] implies that such a tableau exists and is unique. This ends the proof.

End of the proof of Theorem 5.5 Let $\lambda$ be a $(n, m)$-admissible partition. Since $\rho \subset \lambda$, Lemma 5.6 implies that the partition $\mu=((\lambda-\rho))$ is $(n, m-1)$-admissible. And by induction, $S_{\mu}$ appears with a non-zero coefficient in $S_{\rho}^{m-1}$. The positivity of the Littlewood Richardson coefficients implies that each partition $\nu$ such that $c_{\mu, \rho}^{\nu} \neq 0$ appears with a non-zero coefficient in the expansion of $S_{\rho}^{m}$. In particular, 
from Lemma 5.7, it is the case for $\lambda$. This shows that $c_{\lambda}^{n, m} \neq 0$ if and only if $\lambda$ is $(n, m)$-admissible and proves the Theorem. $\square$.

Note that other expansions of Macdonald functions can be found in the literature (for example HallLittlewood polynomials can be expanded in terms of plane partitions [17]), it should be interesting to investigate the properties of $\mathfrak{D}_{k}(\mathbb{X} ; q)$ which can be deduced from these expansions.

\section{Acknowledgments}

We are grateful to Alain Lascoux for fruitful discussions.

\section{References}

[1] G. E. Andrews, MacMahon's partition analysis. I. The lecture hall partition theorem, Mathematical essays in honor of Gian-Carlo Rota (Cambridge, MA, 1996), Progr. Math., vol. 161, Birkhuser Boston, Boston, MA, 1998, pp. 1-22.

[2] H. Belbachir, A. Boussicault and J.-G. Luque, Hankel hyperdeterminants, rectangular Jack polynomials and even power of the Vandermonde, 2007, arXiv:0709.3021.

[3] A.D. Berenstein and A.V. Zelevinsky, Tensor product multiplicities and convex polytopes in partition spaces, J. Algebr. Comb. 1, 1992, 7-22.

[4] A. Cayley, On the theory of determinants, Transaction of the Cambridge Philosophical Society, 8, 1843, 1-16.

[5] A. Cayley, Mémoire sur les hyperdéterminants, Journal für die reine und angewandte Mathematik, 30, 1846, 1-37.

[6] A. Debiard, Polynômes de Tchébychev et de Jacobi dans un espace euclidien de dimension p, C.R. Acad. Sci. Parsi (sé. I), 296, 1983, 529-532.

[7] P. Di Francesco, M. Gaudin, C. Itzykson and F. Lesage, Laughlin's wave functions, Coulomb gases and expansions of the discriminant, Int. J. Mod. Phys. A 9, 1994, 4257-4351.

[8] J. Haglund, M. Haiman and N.Loehr, A Combinatorial Formula for Macdonald Polynomials, J. Amer. Math. Soc. 18, 2005, 735-761.

[9] H. Jack, A class of symmetric polynomial with a parameter, Proc. R. Soc. Endinburgh (A), 69, 1970, 1-18.

[10] H. Jack, A surface integral and symmetric functions, Proc. R; Soc. Edinburgh (A), 69, 1972, $347-63$.

[11] J. Keneko, Selberg integrals and hypergeometric functions associated with Jack polynomials, S.I.A.M. Journal Math. Analysis, 24, 1993, 1086-1110.

[12] R.C. King, F. Toumazet, B.G. Wybourne, The square of the Vandermonde determinant and its $q$ generalization, J. Phys. A: Math. Gen. 37, 2004, 735-767.

[13] A. Korányi, Hua-type integrals, hypergeomatric functions and symmetric polynomials, Proceeding of a Conference in Memory of L K Hua, Beijin, 1998. 
[14] R. B. Laughlin ,Anomalous Quantum Hall Effect: An Incompressible Quantum Fluid with Fractionally Charged Excitations, Phys. Rev. Lett. 50, 1983, 1395-1398

[15] L. Lapointe, A. Lascoux and J. Morse, Determinantal expression and recursion for Jack polynomials, Electon. J. Combin. 1, 2000, 7pp.

[16] L. Lapointe, A. Lascoux and J. Morse, Determinantal expressions for Macdonald polynomials, International Mathematics Research Notices, 1998, n 18.

[17] A. Lascoux, Adding \pm 1 to the argument of a Hall-littlewood polynomial, Seminaire Lotharingien de Combinatoire 54, 2005, 17pp electronic.

[18] A. Lascoux, Symmetric function and combinatorial operators on polynomials, CBMS 99, American Mathematical Society, 2001.

[19] A. Lascoux, Gaudin functions, and Euler-Poincaré characteristics, arXiv:0709.1635.

[20] J.-G. Luque and J.-Y. Thibon, Hankel hyperdeterminants and Selberg integrals, J. Phys. A: Math. Gen., 36, 2003, 5267-5292.

[21] J.-G. Luque and J.-Y. Thibon, Hyperdeterminantal calculations of Selberg's and Aomoto's integrals, Molecular Physics, 10-20 June 2004, Vol. 102, No 11-12, 2004, 1351-1359.

[22] S. Matsumoto, Two parameters circular ensembles and Jacobi-Trudi type formulas for Jack functions of rectangular shape, arXiv:math.PR/0608751 v1, 2006.

[23] I. G. Macdonald, Symmetric functions and Hall polynomials, second edition, Oxford University Press Inc., New York, 1995.

[24] L.H. Rice, P-way determinants with an application to transvectants, American Journal of Mathematics, 40, 1918, 242-262.

[25] T. Scharf, J.-Y. Thibon and B.G. Wybourne, Powers of the Vandermonde determinant and the quantum Hall effect, J. Phys. A.: Math. Gen., 27, 1994, 4211-4219.

[26] J. Sekiguchi, Zonal spherical functions on some symmetric spaces, Pibl. RIMS, Kyoto Univ., 12, 1977, 455-459.

[27] A. Selberg, Bemerkninger om et multiplet integral, Norsk Mathematisk Tidsskrift, 26, 1944, 71-78.

[28] N. P. Sokolov, Spatial matrices and their applications (in Russian), Gosudarstv. Izdat. Fiz.-Mat. Lit., Moscow, 1960.

[29] O. Warnaar, q Selberg integrals and Macdonald polynomials, Ramanujan J. 10 2, 2005, 237-268. 